\title{
BAYESIAN VISION FOR SHAPE RECOVERY
}

\author{
$\underline{\text { André Jalobeanu }}$ \\ USRA/RIACS \\ NASA Ames Research Center MS 269-4 \\ Moffett Field CA 94035-1000, USA \\ email: ajalobea@riacs.edu
}

\begin{abstract}
We present a new Bayesian vision technique that aims at recovering a shape from two or more noisy observations taken under similar lighting conditions. The shape is parametrized by a piecewise linear height field, textured by a piecewise linear irradiance field, and we assume Gaussian Markovian priors for both shape vertices and irradiance variables. The observation process, also known as rendering, is modeled by a non-affine projection (e.g. perspective projection) followed by a convolution with a piecewise linear point spread function, and contamination by additive Gaussian noise. We assume that the observation parameters (e.g. camera pose and noise variance) are calibrated beforehand.

The major novelty of the proposed method consists of marginalizing out all the nuisance parameters (such as the irradiance field and the prior model hyperparam-. eters), which is achieved by Laplace approximations. This reduces the inference to minimizing an energy that only depends on the shape vertices; : and therefore allows an efficient Iterated Conditional Mode (ICM) optimization scheme to be implemented. A Gaussian approximation of the posterior shape density is computed, thus providing not only an estimate of the mean geometry, but also of the uncertainty, which enables us to build a recursive algorithm to easily incorporate new data into the system.

We illustrate the effectiveness of the general method described here by shape reconstruction results in a $2 \mathrm{D}$ case. A $3 \mathrm{D}$ version using the exact same approach is currently under development and aims at recovering a surface from stereo pairs or multiple images, directly reconstructing the topography by marginalizing out both albedo and shading effects.
\end{abstract}

Key Words: stereo vision, shape reconstruction, marginalization, uncertainty estimation, rendering 


\title{
Bayesian Vision for Shape Recovery
}

\author{
André Jalobeanu \\ USRA / RIACS \\ NASA Ames Research Center MS 269-4 \\ Moffett Field CA 94035-1000, USA \\ email: ajalobealriacs.edu
}

\begin{abstract}
We present a new Bayesian vision technique that aims at recovering a shape from two or more noisy observations taken under similar lighting conditions. The shape is parametrized by a piecewise linear height field, textured by a piecewise linear irradiance field, and we assume Gaussian Markovian priors for both shape vertices and irradiance variables. The observation process, also known as rendering, is modeled by a non-affine projection (e.g. perspective projection) followed by a convolution with a piecewise linear point spread function, and contamination by additive Gaussian noise. We assume that the observation parameters are calibrated beforehand.

The major novelty of the proposed method consists of marginalizing out the irradiances considered as nuisance parameters, which is achieved by Laplace approximations. This reduces the inference to minimizing an energy that only depends on the shape vertices, and therefore allows an efficient Iterated Conditional Mode (ICM) optimization scheme to be implemented. A Gaussian approximation of the posterior shape density is computed, thus providing estimates both the geometry and its uncertainty. We illustrate the effectiveness of the new method by shape reconstruction results in a $2 \mathrm{D}$ case. A $3 \mathrm{D}$ version is currently under development and aims at recovering a surface from multiple images, reconstructing the topography by marginalizing out both albedo and shading.
\end{abstract}

\section{INTRODUCTION}

In this work, we investigate the possibility of recovering a shape from a set of corrupted observations. To validate the proposed approach, we define the problem in a $2 \mathrm{D}$ space and perform experiments in this space. Once the approach has been clearly defined, and the model and algorithm choice justified (at least experimentally), the idea is to extend it to the $3 \mathrm{D}$ framework, more realistic. The final goal will be to recover a $3 \mathrm{D}$ surface geometry from multiple 2D images, in an efficient and robust way, without having to also infer the lighting and the spatially variable reflectance properties of this surface, which should be treated as nuisance parameters.

In the plane, the problem is defined as follows. The surface is a Lambertian emitter defined by a finite curve parametrized by a set of $N_{v}$ vertices $\mathbf{v}=\left\{\mathbf{v}^{k}\right\}$, and has an irradiance field attached to it, parametrized by a set of irradiance variables $L=\left\{L^{j}\right\}$. There are at least 2 pinhole cameras (parametrized by the set $\Theta=\left\{\Theta^{i}\right\}$ : position, orientation, etc.) that record an intensity signal after projecting the irradiance onto a segment (in 3D it would be a perspective projection), see Fig. 1. The intensity is sampled after convolution with a sampling kernel, or point spread function (PSF), denoted by $h$. Then it is comupted by a white additive Gaussian noise. Our goal is to provide an estimate of the geometry as well as the uncertainty related to this estimate. 

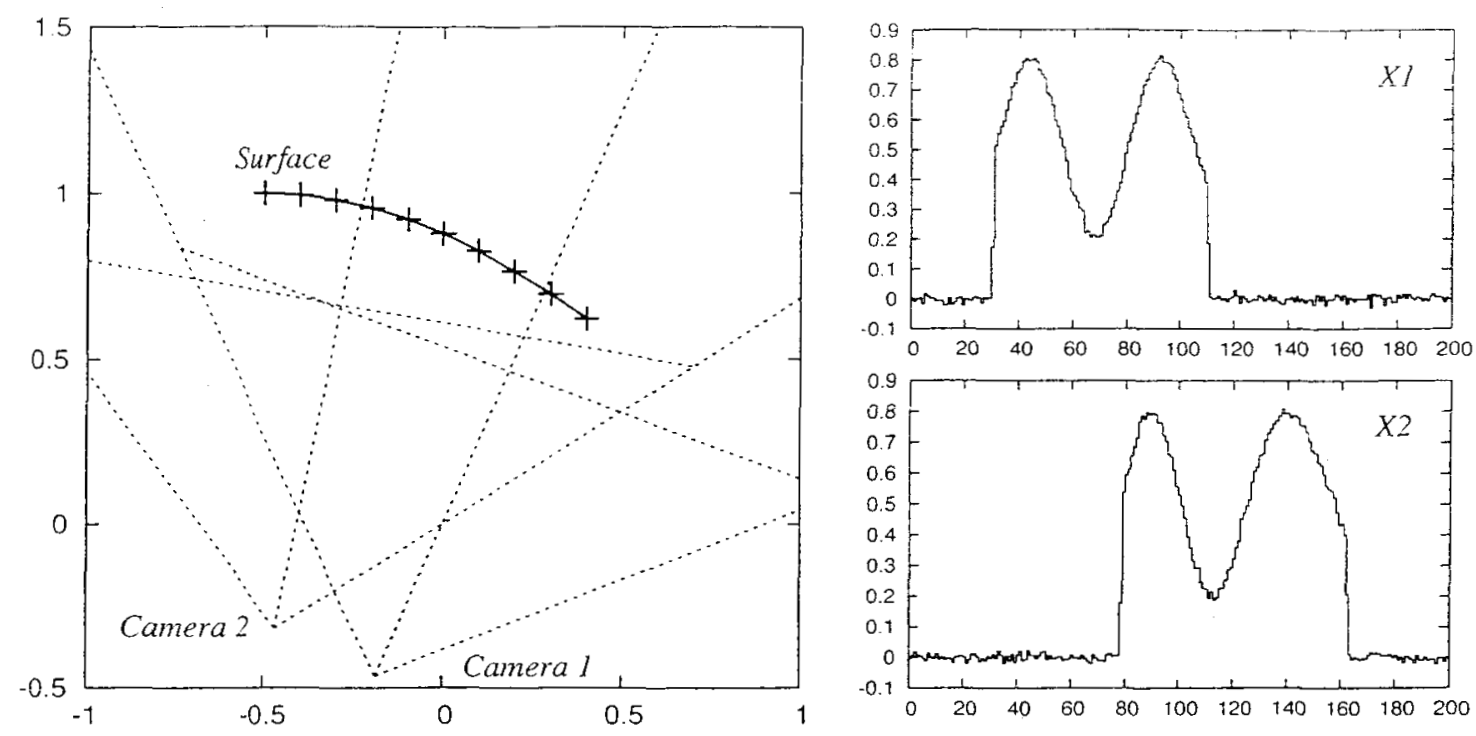

FIGURE 1. Left: the geometry configuration in the object space, showing the 2 cameras. Right: the intensity recorded by each camera. The irradiance field is not shown here (we choose a smooth sine function with values between 0.2 and 0.8 ).

To address this problem, we use a Bayesian framework [2]. To simplify it and get an efficient and stable inference procedure, we make a few assumptions about the lighting scheme. We assume similar lighting conditions (as in a stereo setting), which enables us to consider a single irradiance field attached to the surface, which acts as a texture which is warped to form the observed intensities. Usually a surface is parametrized by an albedo field $\rho$ and a reflectance function $f$, and the irradiance $L$ is such that $L \propto \rho f$. If we assume that we have similar lighting conditions between images, and that $f$ does not depend on the angle between surface and camera, then $f$ is constant from one image to another (though spatially variable), therefore we can re-parametrize by the irradiance.

We can summarize our contributions as follows. First, we derive a model of the unknown surface by choosing an appropriate parametrization and efficient priors to stabilize the solution. Second, we show how to choose an appropriate discretization scheme by understanding the image formation process. Finally, as mentioned above, irradiances are used as parameters. We treat them as nuisance parameters and marginalize them out, deriving efficient approximations to remain computationally tractable.

\section{THE FORWARD PROBLEM}

\subsection{Generative model and posterior distribution}

We assume that all the parameters are random variables governed by a joint probability distribution. The relationships between all these variables are given as a graphical model in Fig. 2 (left), where each arrow represents a conditional density, and each leaf 
node a prior density. If an initial surface estimate is provided, the surface model is then given by this initial estimate and its uncertainty. Otherwise, if we have little knowledge about the surface geometry, we will just assume a smoothness prior. In all cases we choose to use a Gaussian distribution. The camera parameters follow a Dirac distribution around the value obtained by calibration (in our experiment, they are assumed known).

The observations are assumed to be independent and corrupted by a zero-mean white Gaussian noise of variance $\sigma^{2}$. Therefore the conditional density of an observation given the surface and camera parameters is an iid Gaussian of variance $\sigma^{2}$ and mean $I(\mathrm{v}, L, \Theta)$. The intensity $I$ is synthesized from the surface $(\mathrm{v}, L)$ using the camera parameters $\Theta$ (this is also known as rendering and it is a deterministic process, described in Section 2.4). The likelihood of both surface and camera parameters is expressed as:

$$
P(X \mid \mathbf{v}, L, \Theta)=\frac{1}{Z_{\sigma}} \epsilon^{-U(\mathbf{v}, L)} \quad \text { where } U(\mathbf{v}, L)=\frac{1}{2 \sigma^{2}} \sum_{p, i}\left(I_{p}\left(\mathbf{v}, L, \Theta^{i}\right)-X_{p}^{i}\right)^{2}
$$
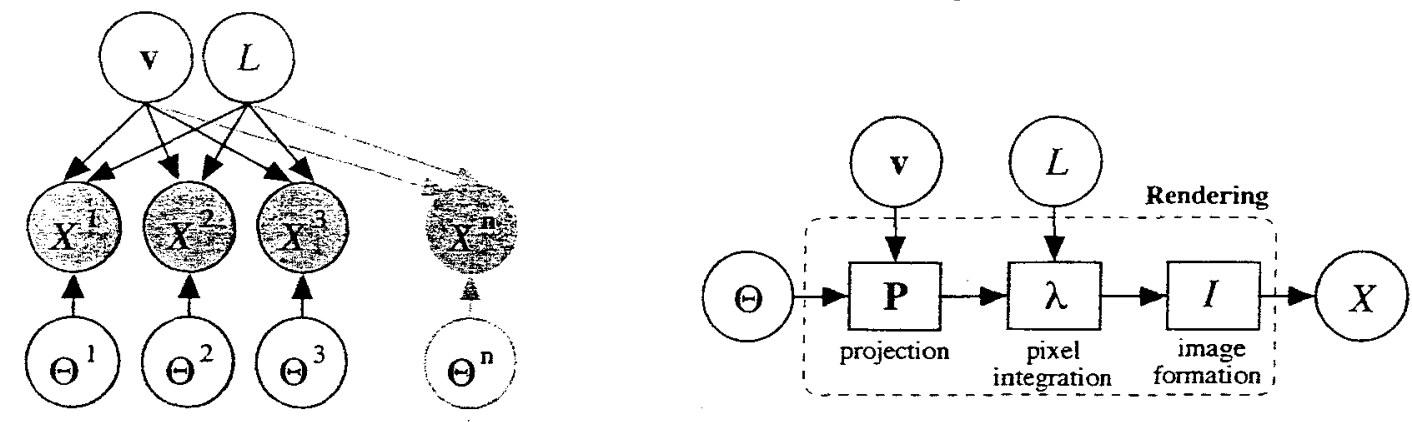

FIGURE 2. The image formation model. Left: the multi-image graphical model. Right: the detailed model for one image (circles and rectangles respectively represent stochastic and deterministic processes).

\subsection{Surface parametrization and topology}

We parametrize the geometry by a set of coarse vertices $\left\{\mathbf{v}^{k}\right\}$ using segments as shown on Fig. 3. To constrain the vertices, we parametrize them by a height field $z(x)$ : we have $\mathrm{v}^{k}=\left(x^{k}, z^{k}\right)$, where the $x^{k}$ form a fixed uniform grid.

Each segment is uniformly subdivided into $N_{s}$ sub-segments containing $N_{s}-1$ aligned fine vertices $\left\{\mathrm{v}^{j}\right\}$ which define the irradiance field. The irradiance has a higher resolution than the geometry. There are multiple ways of choosing the irradiance model: it can be piecewise constant between the fine vertices ( $L^{j}$ lies between 2 fine vertices), or piecewise linear $\left(L^{j}\right.$ is defined on the vertex $\left.v^{j}\right)$, as illustrated by Fig. 3. We will explain in Section 2.4 why a piecewise linear irradiance is preferred.

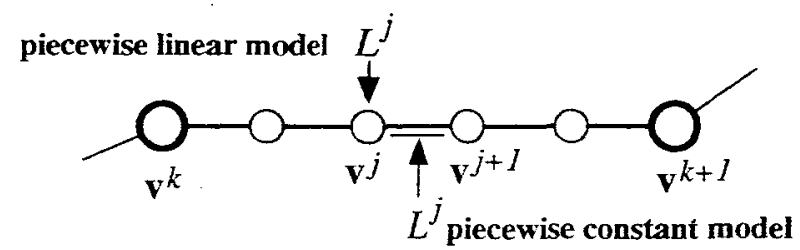

FIGURE 3. The geometry parametrization and topology (subdivided segments), and the 2 possible irradiance models (piecewise linear and piecewise constant). 
linear models for both irradiance and sampling is the only way to get a smooth energy function, with continuous derivatives. For non-boundary vertices, linear irradiance seems to be sufficient: however if we consider vertices located at object boundaries for occlusion boundaries), the model is discontinuous so we need a continuous sampling scheme to achieve the desired smoothness.

Why is this smoothness so important? Because any deterministic optimization algorithm has little chance to converge to the global optimum if there are discontinuities in the derivatives (on the left figure, the local minima are quite obvious!). For computational reasons, we do not intend to use stochastic techniques such as simulated annealing to get around local minima problems.
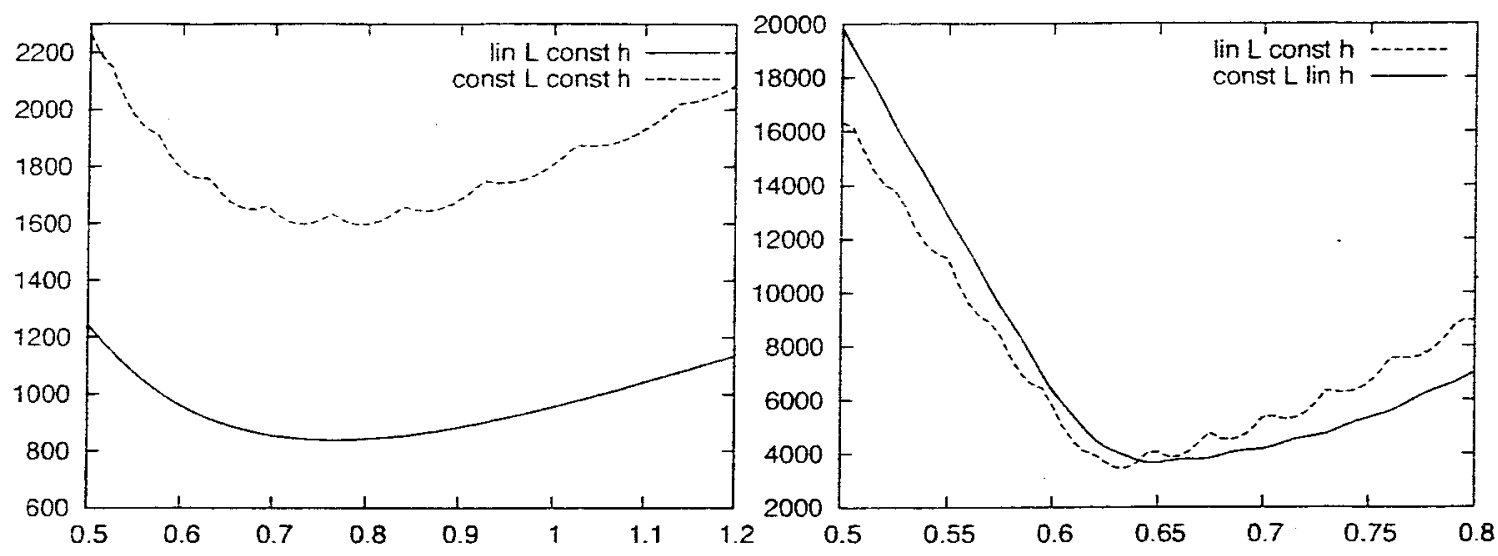

FIGURE 4. Variation of the - $\log$ likelihood $(U+$ const.) as a function of one of the vertices (fixed irradiance). Left: non-boundary vertex, piecewise linear vs. piecewise constant irradiance model $L$. Right: boundary vertex, piecewise linear vs. piecewise constant sampling kemel $h$.

\section{THE INVERSE PROBLEM: BAYESIAN INFERENCE}

Computer vision, or model reconstruction from observations, can be seen as the inverse problem of rendering. Bayesian inference [2] is an efficient way to deal with such ill-posed inverse problems. In the Bayesian framework, model recovery becomes a parameter estimation problem (more precisely, we estimate parametric pdfs), which is achieved by using existing efficient optimization algorithms.

Using Bayes' rule and the graphical model of Fig. 2 (left) we get the joint posterior:

$$
P\left(\mathbf{v}, L,\left\{\Theta^{i}\right\} \mid\left\{X^{i}\right\}\right) \propto P(\mathbf{v}, L) \prod_{i} P\left(X \mid \mathbf{v}, L, \Theta^{i}\right) P\left(\Theta^{i}\right)
$$

It is well-known in Bayesian inference that one should integrate the posterior over all unwanted, or nuisance parameters [1], to achieve a good stability: in our case, the camera parameters and the irradiances should be marginalized out. Stability is not the only reason to perform marginalization, as we will see in the algorithm section. Camera marginalization is rather simple since we assumed a Dirac distribution for $\Theta$. 


\subsection{The geometry and irradiance priors}

To stabilize the solution, in our 2D experiments we use very simple and spatially invariant smoothness priors on both heights and irradiances, corresponding to first order Markov chains (limited to nearest neighbor interactions):

$$
\begin{array}{ll}
P(\mathbf{v})=\frac{1}{Z_{\alpha}} e^{-\alpha \Phi(\mathbf{v})} & \text { where } \Phi(\mathbf{v})=\sum_{k}\left(z^{k+1}-z^{k}\right)^{2} \\
P(L)=\frac{1}{Z_{\beta}} e^{-\alpha \Phi(L)} & \text { where } \Phi(L)=\sum_{j}\left(L^{j+1}-L^{j}\right)^{2}
\end{array}
$$

More complex priors should be used if spatial adaptivity is required, or if discontinuities need to be modeled. Using efficient priors is important when dealing with missing or insufficient data (in real scenarios, some parts of the surface may be hidden) [4].

\subsection{The deterministic image formation process}

We focus here on the rendering process, or how to obtain discrete pixel intensities from the set of vertices and irradiances in several steps (see Fig. 2 right). Each vertex is projected according to the following non-linear pinhole camera model (this is equivalent to a perspective projection in $3 \mathrm{D}$ ), where $\mathrm{u}$ is the direction, $\mathrm{T}$ the location and $a, b$ constants related to internal camera parameters:

$$
\mathbf{P}_{x}^{j}=a \frac{x^{j} \mathbf{u}_{z}-z^{j} \mathbf{u}_{x}+\mathbf{T}_{x}}{x^{j} \mathbf{u}_{x}+z^{j} \mathbf{u}_{z}+\mathbf{T}_{z}}+b \quad \text { with } \quad \Theta=\{\mathbf{u}, \mathbf{T}, F\}
$$

If we have a linear irradiance model between fine vertices, the projected irradiance field $\mathcal{L}$ is assumed to be also linear, since the fine segments are small enough. The intensity for each pixel $I_{p}$ is obtained by first convolving $\mathcal{L}$ with a PSF $h$, then point sampling on a regular grid $\{p\}: I_{p}=(\mathcal{L} \star h)(p)$.

The PSF can be decomposed as a discrete sum of translated sampling kernels $\Lambda$, so that $I_{p}$ is obtained by convolution with a fixed $\Lambda$, sampling and then a discrete convolution of the pixel values which can be taken out of the rendering. For simplicity, we ignore this last convolution and assume that $h=\Lambda$.

Since $\mathcal{L}$ is a linear function of $\left\{L^{j}\right\}$, we rewrite the imaging equation as follows:

$$
I_{p}=\sum_{j} \lambda_{p}^{j} L^{j} \quad \text { where } \quad \lambda_{p}^{j}=\text { function of } \mathrm{P}(\mathbf{v}, \Theta)
$$

Then an important question arises: what kernel should we use? The simplest would be a normalized box function $h(x)=1$ with $x \in[-0.5,0.5]$. However, we might need a more continuous one, such as the hat function $h(x)=1-|x|$ with $x \in[-1,1]$.

We have implemented the 4 possible combinations of piecewise linear and constant models for both irradiance and sampling kernel, and studied the behavior of the energy $U(\mathbf{v}, L, \Theta)$ relative to one of the images, when one of the vertex heights $z^{k}$ varies (all others being equal to their true value). Fig. 4 clearly illustrates the fact that choosing 


\subsection{Irradiance marginalization, approximations}

We need to calculate the following integral to marginalize out the irradiance:

$$
P\left(\mathbf{v} \mid\left\{X^{i}\right\}\right)=\int_{\Omega} P\left(\mathbf{v}, L \mid\left\{X^{-i}\right\}\right)=P(\mathbf{v}) \int_{\Omega} e^{-U(\mathbf{v}, L)-\beta \Phi(L)} d L
$$

which can be achieved by using a Laplace approximation, assuming that the integrand can be well approximated by a multivariate Gaussian distribution. The integrand is proportional to the posterior $P\left(L \mid v,\left\{X^{i}\right\}\right)$ (fixed vertices), which is a Gaussian distribution if we assume unbounded irradiances (since $I_{p}$ is linear in $L, U$ is quadratic in $L$, and we also have a quadratic penalty $\Phi$ ). For physical reasons, the irradiance is positive and bounded, so the distribution is not rigorously Gaussian. We will assume that the data constrains it to take values far enough from the bounds to ensure the validity of the Laplace approximation. Now we need to calculate the determinant of the covariance matrix $[\Xi]$, and the optimum (MAP) of $L$ given the current geometry v, so that:

$$
\begin{array}{r}
P\left(\mathbf{v} \mid\left\{X^{i}\right\}\right) \propto P(\mathbf{v}) \frac{1}{\sqrt{|\Xi|}} e^{-U(\mathbf{v}, \hat{L})-\beta \Phi(\hat{L})} \\
\hat{L}(\mathbf{v})=\arg \max _{L} P\left(L \mid \mathbf{v},\left\{X^{i}\right\}\right)=\arg \min _{L}[U(\mathbf{v}, L)-\beta \Phi(L)] \\
{\left[\Xi^{-1}\right]_{i j}=\frac{\partial^{2}}{\partial L^{i} \partial L^{j}}[U(\mathbf{v}, L)-\beta \Phi(L)]_{\hat{L}(\mathbf{v})}}
\end{array}
$$

Experiments have shown that the $-\log |\Xi|$ compared to the term $U(\mathbf{v}, \hat{L})+\beta \Phi(\hat{L})$ has negligible variations when changing vertex heights, so that it can be neglected when optimizing w.r.t. vertices (see Fig. 5 for an illustration).

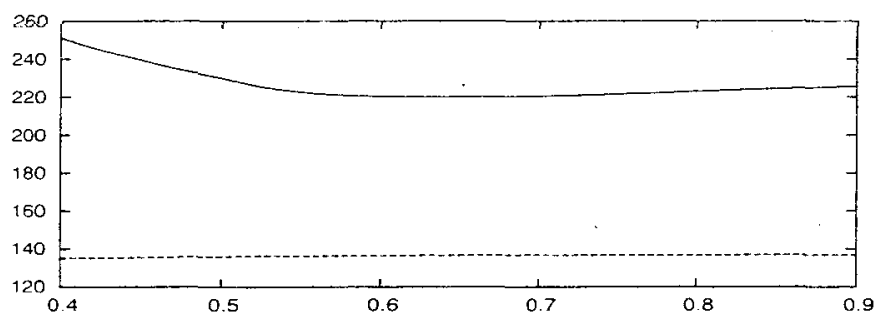

FIGURE 5. Solid line: marginal energy $U^{\prime}$ as a function of one of the vertices, computed with the proposed approximation; Dashed line: the normalizing constant that was neglected in this computation.

The optimum can be computed by a diagonal Newton-Raphson descent algorithm; a few steps are sufficient to obtain a good convergence (see Section 3.2 for derivative computations). However, it is preferable to have a closed-form approximation of this optimum, so that the derivatives can be calculated as it will be mentioned in Section 3.2. We propose one that has a reasonable accuracy when the weights $\lambda$ are close to 0 or 1 and there is little regularization. This gives good results in general, despite the absence of regularization, making the optimization itself useless in practice:

$$
\hat{L}^{j}(\mathrm{v}) \simeq \frac{\sum_{i, p}\left(\lambda_{p}^{j}\right)^{i} X_{p}^{i}}{\sum_{i, p}\left(\lambda_{p}^{j}\right)^{i}}
$$


Finally we propose the following approximation of the marginalized posterior, so that the entire problem reduces to minimizing the marginal energy $L^{\prime \prime}(\mathrm{v})$ :

$$
P\left(\mathbf{v}\left\{\left\{X^{i}\right\}\right) x e^{-U^{\prime \prime}(\mathbf{v})} \text { where } U^{-1}(\mathbf{v})=U(\mathrm{v}, \hat{L}(\mathbf{v}))+\beta \Phi(\hat{L}(\mathbf{v}))-\log P(\mathbf{v})\right.
$$

We verify once again that we have made the right choice regarding both irradiance and sampling models: on Fig. 6 the oscillations related to piecewise constant models become quite obvious, and often lead to local minima, and in some cases a bias is noticeable (the global optimum is not the closest to the true value). For boundary vertices the linear sampling scheme is the only one to provide an acceptable marginal energy function.
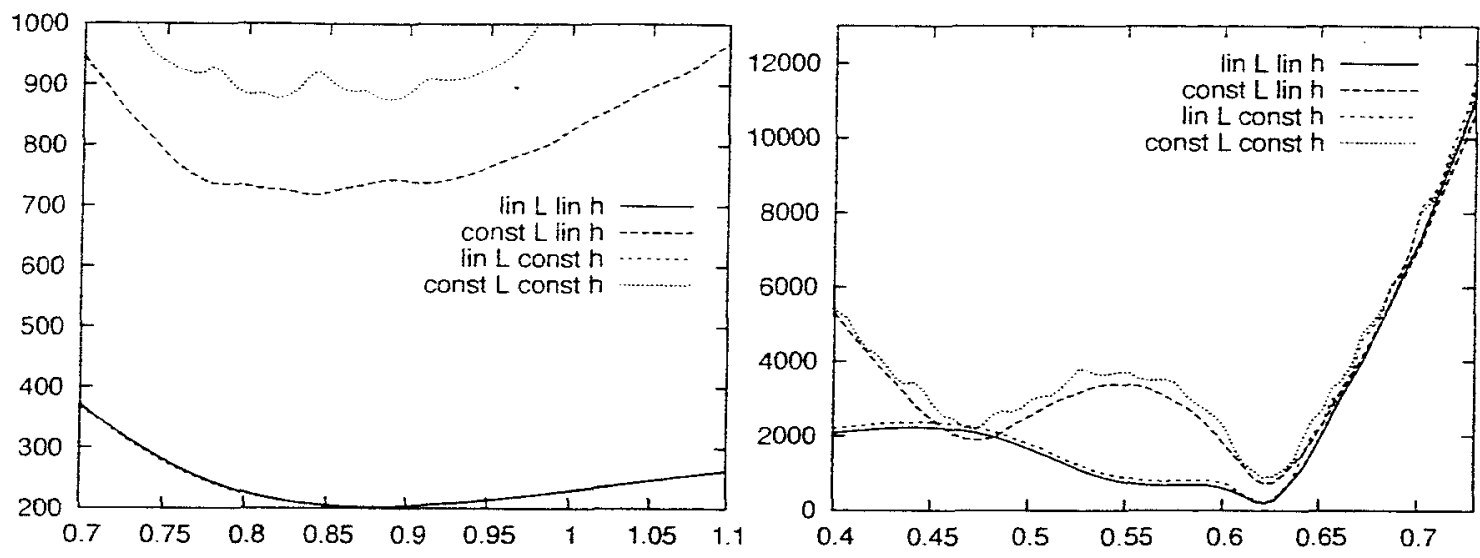

FIGURE 6. Variation of the marginal energy as a function of one of the vertices. Left: non-boundary vertex, piecewise linear vs. piecewise constant irradiance model. Right: boundary vertex, piecewise linear vs. piecewise constant sampling kemel $h$.

\subsection{Computing the derivatives}

In order to achieve surface recovery via deterministic minimization of the marginal energy $U^{\prime}(\mathbf{v})$, we need to compute the derivatives of this energy w.r.t. vertices. This requires us to compute the derivatives of the rendered intensity, of the optimal irradiance and of the prior penalty terms. To do this, we use the chain rule, accounting for the graph structure of all variables involved. For instance, let a vector $\mathrm{C}$ be a function of $\mathrm{A}$ through a set of variables $\left\{\mathbf{B}^{i}\right\}$; then the derivative matrices obey the chain rule:

$$
\left[\frac{\partial \mathbf{C}}{\partial \mathbf{A}}\right]=\sum_{i}\left[\frac{\partial \mathbf{C}}{\partial \mathbf{B}^{i}}\right]\left[\frac{\partial \mathbf{B}^{i}}{\partial \mathbf{A}}\right]
$$

For the rendered intensity, given the graph structure in Fig. 2 (right) we get the following expression when the irradiance is a function of the vertices:

$$
\left[\frac{\partial I_{p}}{\partial \mathbf{v}^{k}}\right]=\sum_{j}\left(\left[\frac{\partial I_{p}}{\partial \lambda_{p}^{j}}\right]\left[\frac{\partial \lambda_{p}^{j}}{\partial \mathbf{P}^{j}}\right]\left[\frac{\partial \mathbf{P}^{j}}{\partial \mathbf{v}^{j}}\right]+\left[\frac{\partial I_{p}}{\partial \hat{L}^{j}}\right]\left[\frac{\partial \hat{L}^{j}}{\partial \mathbf{v}^{j}}\right]\right)\left[\frac{\partial \mathbf{v}^{j}}{\partial \mathbf{v}^{k}}\right]
$$

This derivative is involved in the first and second derivatives of the marginal energy: 


$$
\begin{gathered}
{\left[\frac{\partial L^{\prime}}{\partial \mathbf{v}^{k}}\right]=\frac{1}{\sigma^{2}} \sum_{i, p}\left(I_{p}^{i}-X_{p}^{-i}\right)\left[\frac{\partial I_{p}^{i}}{\partial \mathbf{v}^{k}}\right]+\alpha\left[\frac{\partial \Phi(\mathbf{v})}{\partial \mathbf{v}^{k}}\right]+\beta \sum_{j}\left[\frac{\partial \Phi(\hat{L})}{\partial \hat{L}^{j}}\right]\left[\frac{\partial \hat{L}^{j}}{\partial \mathbf{v}^{k}}\right]} \\
{\left[\frac{\partial^{2} U^{\prime}}{\partial \mathbf{v}^{k} \partial \mathbf{v}^{l}}\right] \simeq \frac{1}{\sigma^{2}} \sum_{i, p}\left[\frac{\partial I_{p}^{i}}{\partial \mathbf{v}^{k}}\right]\left[\frac{\partial I_{p}^{i}}{\partial \mathbf{v}^{l}}\right]+\alpha\left[\frac{\partial^{2} \Phi(\mathbf{v})}{\partial \mathbf{v}^{k} \partial \mathbf{v}^{l}}\right]+\beta \sum_{j}\left[\frac{\partial^{2} \Phi(\hat{L})}{\partial \mathbf{v}^{k} \partial \hat{L} j}\right]\left[\frac{\partial \hat{L}^{j}}{\partial \mathbf{v}^{l}}\right]}
\end{gathered}
$$

where $I_{p}^{i}$ refers to the $i$-th intensity image rendered with $\Theta^{i}$. We approximate the second derivatives of $U^{\prime}$ by neglecting the contribution of the second derivatives of the intensity and the optimal irradiance. Here we can see that we also need to compute the derivative of the optimal irradiance, hence the advantage of having a closed-form expression (function of v) such as the one in Eqn. (11).

\subsection{The surface recovery algorithm}

The goal of the reconstruction algorithm is to provide a Gaussian approximation of the posterior marginal $\left.P(\mathrm{v}\}\left\{X^{i}\right\}\right)$. To achieve this, we need to compute the mode $\hat{\mathrm{v}}$ of this distribution, which is equivalent to minimizing the energy $U^{\prime}$ w.r.t. the geometry. Then we need a quadratic approximation of the energy around the optimum, hence the second derivatives (16) taken at $\hat{\mathbf{v}}$. The covariance matrix $[\Sigma]$ provides an measure of the uncertainty on the geometry estimate $\hat{\mathrm{v}}$ :

$$
\left[\Sigma^{-1}\right]=\left[\frac{\partial^{2} U^{\prime}}{\partial \mathbf{v}^{k} \partial \mathbf{v}^{l}}\right]_{\hat{v}}
$$

Keeping the inverse covariance matrix at the end of the reconstruction enables us to build a recursive update algorithm, by using this Gaussian approximation as a prior for the next surface estimation process. This way, data can be added to the model sequentially, which can have multiple advantages. For instance the computational complexity is reduced since we do not need to use all the data at the same time. Furthermore, we can remove the restrictive assumptions about the lighting, by processing sets of images with similar lighting conditions simultaneously, and combining the different sets recursively.

The proposed optimization algorithm is iterative, and at each step the rendering process is linearized around the current estimate $\tilde{\mathrm{v}}$ using the intensity derivatives:

$$
I_{p}(\mathbf{v}, \hat{L}, \Theta) \simeq I_{p}(\tilde{\mathbf{v}}, \hat{L}(\tilde{\mathbf{v}}), \Theta)+\sum_{k}\left[\frac{\partial U^{\prime}}{\partial \mathbf{v}^{k}}\right]\left(\mathbf{v}^{k}-\tilde{\mathbf{v}}^{k}\right)
$$

This makes $U(\mathrm{v}, \hat{L})$ a quadratic form in $\mathrm{v}$. Since we choose quadratic penalty functions for the priors, $U^{\prime}$ is also quadratic in v. Moreover, it has a first order Markov structure when there are no occlusions (the dependence is limited to the first order neighbors). Therefore, optimizing this quadratic form is best achieved by an Iterative Conditional Mode (ICM) procedure, which benefits from the local dependence structure. Because of the very limited dependence, using a conjugate gradient in this case is clearly not a good choice, as our experiments have shown. In practice, given the weak dependence between vertices, an independent optimization turned out to be very efficient, and was achieved by using a diagonal quasi-Newton descent technique: 


$$
\hat{\mathbf{v}}^{k}-\hat{\mathrm{v}}^{k}-\left[\frac{\partial U^{\prime \prime}}{\partial \mathbf{v}^{k}}\right]\left[\frac{\partial^{2} L^{\prime \prime}}{\partial\left(\mathbf{v}^{k}\right)^{2}}\right]^{-1}
$$

We also noticed that an accurate optimization of the quadratic form does not help increase the computation speed nor the result quality, since linearization is only a rough approximation. We did not observe any improvement from one to multiple iterations, therefore we do not recommend more than one step of descent before recomputing the local quadratic form around the updated geometry estimate.

The proposed algorithm is summarized as follows:

- Input: $\left\{X^{i}\right\},\left\{\Theta^{i}\right\}, \sigma^{2}$.

- Initialization: $\tilde{\mathrm{v}}=$ constant (or a previous estimate if available);

- Repeat until convergence:

- Irradiance marginalization: compute $\hat{L}(\tilde{\mathbf{v}})$, Eqn. (11);

- Intensity and irradiance derivatives: Eqn. (14), derivative of Eqn. (11);

- First and second derivatives of $U^{\prime}$ : Eqns. (15)-(16);

- One step diagonal quasi-Newton update: Eqn. (19).

- Inverse covariance estimation: Eqn. (17).

- Output: geometry $\hat{\mathbf{v}}=\tilde{\mathbf{v}}$, covariance $\left[\Sigma^{-1}\right]$.

The Bayesian approach enables us to automatically estimate the regularization parameters $\alpha$ and $\beta$, as well as the subdivision level $N_{s}$ (i.e. model selection, via Bayes factors). This has been investigated in our experiments, but it is not described in this paper. Moreover, the segment subdivision level could be selected locally and dynamically (in the inner loop of the algorithm), depending on the current local geometry.

\section{2D EXPERIMENT: RESULTS AND CONCLUSIONS}

The reconstruction algorithm has been successfully applied to the problem described in the introduction, and the results are shown on Fig. 7. We have $\sigma=0.01$ in this experiment (1\% of the intensity range, since $I \in[0,1]$ ). The reader can evaluate the reconstruction quality by observing the estimated vertices and the error bars (corresponding to a marginal posterior probability greater than 0.1 , computed using the estimated inverse covariance). The plot on the right shows the joint distribution of 3 pairs of vertices conditioned upon all others: only nearest neighbors interact and can therefore be strongly correlated (the correlation decays exponentially with the distance). The coarse segments are larger than pixels, otherwise there would be longer range interactions. This means that the inverse covariance matrix is sparse: it is possible to store it along with the surface estimate and to use it as a prior for subsequent inferences.

The main conclusions of our 2D experiments are: 1) continuous models for both irradiance and sampling should be used to ensure the continuity of the energy functions; 2) irradiance marginalization reduces the problem dimension from $\left(N_{s}+1\right) N_{v}$ irradiances+vertices to $N_{v}$ vertices, also strongly reducing the interaction structure; 3) we observed that marginalization also makes the energy landscape more quadratic, making the use of Newton-like techniques appropriate. 

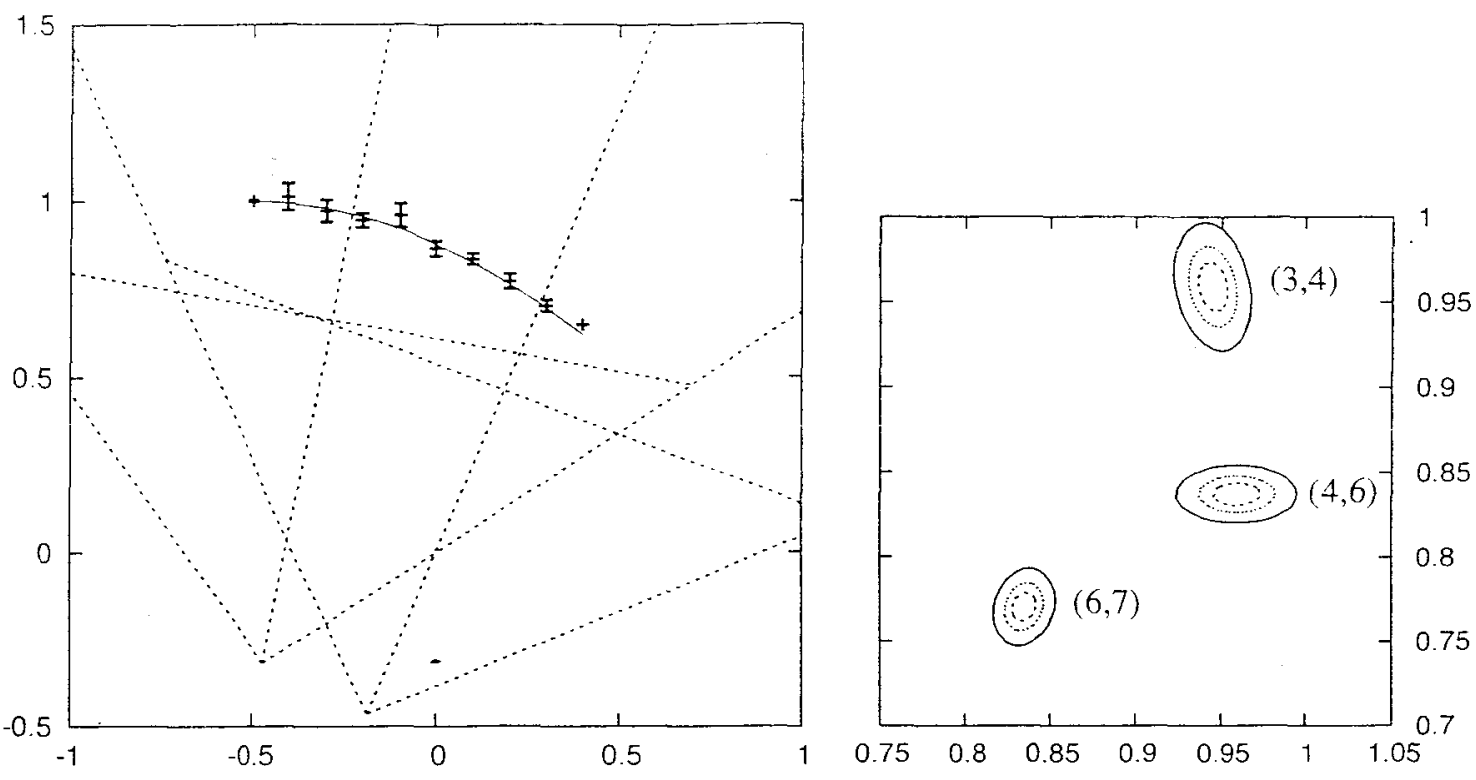

FIGURE 7. The inferred vertex heights ( 10 iterations, parallel quasi-Newton optimization). Left: error bars $=$ inferred geometry (probability $>0.1$ ), solid line = ground truth, dashed lines = the two camera configurations. Right: joint conditional posterior distribution of 2 vertex heights given all others (the indices are shown), illustrating the pairwise interactions.

\section{EXTENSION TO 3D AND FUTURE WORK}

To extend this promising approach to a more useful 3D framework, we use subdivided triangular meshes instead of subdivided segments, and 2D images instead of 1D signals. Hidien surface removal needs to be accurately performed (we assumed there were no occlusions in the $2 \mathrm{D}$ experiments). This is achieved through a recursive polygonal approach which subtracts triangles from an triangle/pixel intersection polygon [4]. Rendering using continuous irradiance and PSF is made possible by computing the order- 2 moments of the visibility polygons [3]. In 3D, the goal is to first infer the scene geometry ( $3 \mathrm{D}$ equivalent of the method described in this paper), then to infer the albedo and reflectance functions using the estimated geometry (empirical Bayes approach).

To achieve accurate surface recovery, designing and studying realistic prior models [4] is needed. Simultaneous reconstruction and camera calibration could also be addressed through marginalization. Finally, Bayesian model selection should be considered if a spatially adaptive mesh subdivision is needed.

\section{REFERENCES}

1. J.O. Berger, B. Liseo, and R. Wolpert. Integrated likelihood methods for eliminating nuisance parameters. Statistical Science, 14(1):1-28, 1999.

2. Jose M. Bernardo and Adrian F. M. Smith. Bayesian Theory. John Wiley \& Sons, Inc., 1994.

3. A. Jalobeanu. Mathematics of accurate super-resolution rendering. RIACS Research Report, in preparation 2004.

4. A. Jalobeanu, F.O. Kuehnel, and J.C. Stutz. Modeling Images of Natural 3D Surfaces: Overview and Potential Applications. In GMBV'04 (part of CVPR'04), Washington, DC, July 2004. 